\title{
Soft X-ray emission from the Low-Mass X-ray Binary Cyg X-2
}

Hirohisa Shirai, Hiromitsu Takahashi, Osamu Nagae and Yasushi Fukazawa

Hiroshima University, Japan

E-mail: shirai@hep01.hepl.hiroshima-u.ac.jp

Suzaku observed results of Cyg X-2 are reported. Cyg X-2 is a Low-Mass X-ray Binary (LMXB) with a weakly-magnetized neutron star (NS) and its luminosity is always close to the Eddington luminosity. Suzaku observed the source in the normal branch (NB) and flaring branch (FB). Seven spectra were extrated according to different positions in the color-color diagram. The continuum of these spectra is moderately well described with the Eastern model, a conbination of soft disk blackbody and hard black body emission. However, all the spectra have broad excess around 1 $\mathrm{keV}$. This excess cannot be explained with the contribution of dust scattering and is thought to be related with the source itself, since comparison of spectra come from including and excluding the central core of the X-ray image does not show any difference in their spectral shape. The broad excess can be represented by either a broad Gaussian with the center energy of $\sim 0.7 \mathrm{keV}$ or a blackbody model with the temperature of $\sim 0.2 \mathrm{keV}$. When the broad Gaussian model is applied to all the 7 spectra, the center energy and width are obtained as almost constant at $0.7 \mathrm{keV}$ and $340 \mathrm{eV}$, respectively, and the equivalent width is $170-300 \mathrm{eV}$ which increases continuously from NB to FB. The equivalent width obtained by Suzaku is much larger than the total of narrow lines detected by previous grating observations. In additon, if the line is assumed to be broad, the estimated line emitting region becomes too close to the NS to prevent ions from the fullyphotoionized plasma. These results indicate that the $1 \mathrm{keV}$ excess cannot be explained by the line components physically. Therefore, we suppose that this excess is represented by the $0.2 \mathrm{keV}$ blackbody component. Such a temperature is lower than neither that of the NS surface $(\sim 2 \mathrm{keV})$ nor the innermost temperature of the accretion disk $(\sim 1 \mathrm{keV})$, the $0.2 \mathrm{keV}$ emission is thought to be from the outer part of the disk. This interpretation is consistent with the picture of a slim disk, where the relative flux from the outer disk (the lower temperature emission) to that from the inner part increases compared with the standard disk.

VII Microquasar Workshop: Microquasars and Beyond

September 1 - 5, 2008

Foca, Izmir, Turkey 


\section{Introduction}

In the high-mass accretion rate close to the Eddington limit, it is predicted that the accretion disk changes its state from the standard one to the slim one. The slim disk relatively increases low temperature radiation as a result of distribution of temperature profile $\left(0.5<p<0.75 ; T^{-p}\right)$ compared with the standard one ( $p=0.75$ ) (Watarai et al. 2000). However, in Galactic Black Hole binaries, the prediction is not well studied, since the number of observations at such high-mass accretion rates is very limited, and the absorption is relatively high. On the other hand, since the number of known weakly-magnetized neutron stars (NSs) is larger than that of Black Holes, there are more low-mass X-ray binaries (LMXBs) which contain NSs and have high-mass accretion rates and lower absorption.

Most luminous LMXBs are called as $\mathrm{Z}$ sources of which the luminosities are always bright and close to the Eddington luminosity $\sim 2 \times 10^{38} \mathrm{erg} / \mathrm{s}$ (Hasinger et al. 1989). Z sources change the states on the timescale of days, and show the $\mathrm{Z}$ shape in their $\mathrm{X}$-ray Color-Color Diagrams (CCDs). The mass accretion rates of the $Z$ sources are thought to increase from the top left to the bottom right of the Z-pattern, that is along to so called horizontal branch (HB), normal branch (NB) and flaring branche (FB). However, the detail physical condition of each branch has not been understood well. Among the $\mathrm{Z}$ sources, Cyg X-2 has relatively small absorption, and it is suitable to observe the emission in the low energy band $(\leq 1 \mathrm{keV})$, where the emission from the accretion disk is dominant. Thus, we through analysis of Cyg X-2 data observed by Suzaku, we study the condition of the accreton disk and the state transition of the $\mathrm{Z}$ sources.

Cyg X-2 is composed of the NS of which the mass is $1.78 \pm 0.23 M_{\odot}$ with the distance of $7.2 \pm 1.1$ kpc (Orosz \& Kuulkers 1999). The orbital period of the binary system is measured as 9.8444 days binary system has an orbital period of 9.8444 days(Casares et al. 1998).

We describe the observation and analysis of the Suzaku data of Cyg X-2 in section 2, and present the results and discussions in section 3 .

\section{Observations and Data Reduction}

We analyzed the Suzaku archival data of Cyg X-2, of which the observation date was on 2006 May 16. Suzaku has instruments suitable to study LMXBs. The X-ray CCD camera, which named as XIS, observe the energy range of $0.2-12 \mathrm{keV}$. Three (XIS 0, XIS 2 and XIS 3) of the four XIS sensors use front-illuminated (FI) CCDs, while XIS 1 utilizes a back-illuminated (BI) one, which has the higher efficiency in the lower energy band. In addition, the Hard X-ray Detector (HXD) has two instruments, HXD-PIN and HXD-GSO, their energy ranges are 10-70 keV and 50-600 keV, respectively. The HXD has no imaging capabirity but unprecedented sensitivity with the lower background above $10 \mathrm{keV}$. Thus, Suzaku can observe the broad band with the high energy resolution.

Cyg X-2 was so bright, that we needed to remove the effect of photon pile-up which affects the spectral shape especially above $8 \mathrm{keV}$. To remove the pile-up effect, we first excluded the circular region of which the radius of 70 pixels (corresponding to 1.2 arcmin) from the centeral core of each XIS image. The other analysis steps were in the standard way.

In order to study the state transition along the shape of the CCD, we extracted light curves of three 
energy bands of $0.5-2 \mathrm{keV}, 4-6 \mathrm{keV}$ and $10-40 \mathrm{keV}$, which are shown in the left panel of 1 . In this observation, it appears that the count rate decreased at short timescales $(\sim 1 \mathrm{ks})$, of which the behaviors we call dips and show the positions with the arrow in the light curves. The intervals between the dips are much shorter than the orbital period of Cyg X-2 (9.8444 days) and thus the dip were not caused by the eclips of the secondary star. So as to trace the state transition, we also extracted the CCD and the Intensity Hard-color Diagram (IHD) from these light curves. As shown in Figure 1 (middle and right panels), Cyg X-2 was the NB and the FB in this observation. Then, seven spectra were extracted according to different positions in the CCD and the IHD, five in the $\mathrm{NB}$, one in the FB and one in the Dip. In the following analysis, we utilized both of FI- and BI-XIS sensors and the HXD-PIN, but the spectra of the BI-XIS are not shown in figures for simplicity.
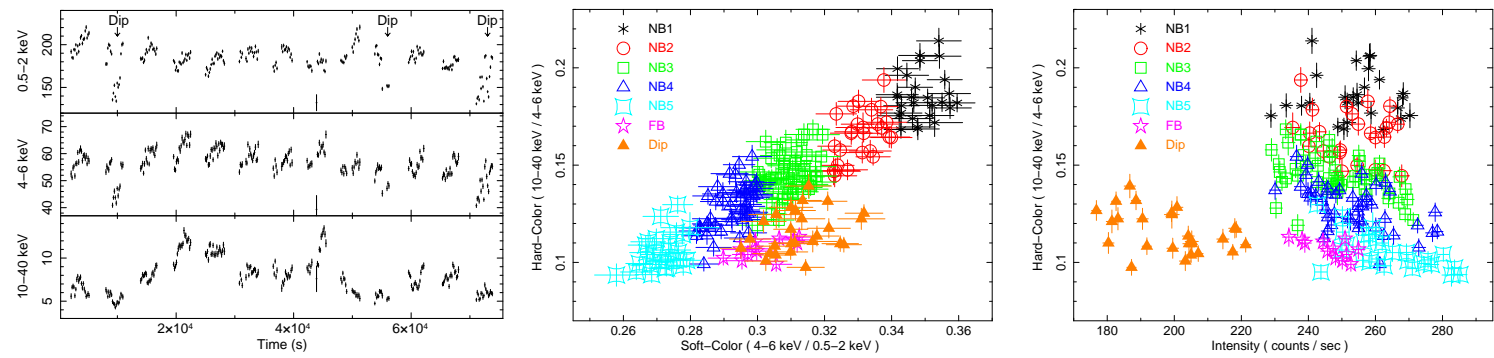

Figure 1: Left: XIS and HXD-PIN light curves of Cyg X-2, where those of the three FI- and the one BI-XIS were summed up into one. Top: $0.5-2 \mathrm{keV}$, middle: $4-6 \mathrm{keV}$ and bottom: $10-40 \mathrm{keV}$. The y-axis is count rate in the unit of counts/s. Middle and right: CCD and IHD of Cyg X-2, respectively, where the light curves of $0.5-2 \mathrm{keV}$ and $4-6 \mathrm{keV}$ are utilized for the soft color and those of 4-6 keV and 10-40 keV are for the hard one.

\section{Results and Discussions}

\subsection{Spectral Variation along the Color-Color Diagram}

Before fitting the spectra, we investigated variation of the spectral shape along the CCD. Figure 2 shows spectral ratios of the five spectra in the NB1, NB3, NB5, FB and Dip to that of the average one. It is clearly seen that the flux below $4 \mathrm{keV}$ continuity increases from NB1 to NB5, while the hard emission above $4 \mathrm{keV}$ decreases. This fact indicates that the spectra compose of two components, the softer one and the harder one. Furthermore, the spectral variation is seen between the NB and the FB, where the FB has the higher flux only in the 4-10 keV band than the NB5. The spectral shape of dip is similar to that of the FB, but the flux decreses $20 \%$

The above spectral variation is consistent with the picture that suggested by Takahashi et al. (2005, 2006). They proposed that the LMXBs spectra can be represented by so-called Eastern model (Mitsuda et al. 1984; Makishima et al. 1989) plus outfbw caused by radiation pressure. The Eastern model is constructed by two components, a Multi-Color Disk (MCD) together with a (Comptnized) blackbody (BB) emission, which represent the emission from the accretion disk and the NS, respectively. Since the luminosity is close to the Eddington limit, it is proposed that some fraction of the matter outfbws, before reaching onto the NS surface, driven by the radiation pressure from the NS surface and the accretion disk. As the mass accretion rate increases, the disk luminosity increases and prevents more matter accreting on the NS surface. This behavior is thought to appear from the 
NB1 to the NB5, where the flux in the softer energy band, which comes from the disk, increases, while that in the harder band, which represents the emission from the NS surface, decreases. In the $\mathrm{FB}$, the amount of the outfbw is supposed to increase more than the NB, and the outfbw itsself becomes optically-thick. We thought that the higher 4-6 keV flux in the FB than the NB caused by the optically-thick outfbw. Furthermore, the Dip state always transits from the FB. This fact indicate that optically-thick outfbw hides the radiation from the central region. In the following, we concentrate the analysis in the soft energy band $(\sim 1 \mathrm{keV})$, and employ the simple Eastern model (not considering the outfbw effect) for simplicity.

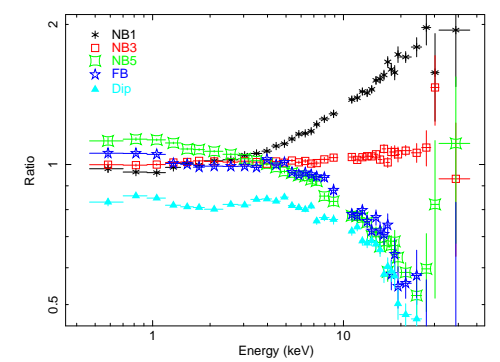

Figure 2: Spectral ratios between the averaged spectrum and 5 spectra in the NB1, NB3, NB5, FB and Dip branches.

\subsection{Broad Structure around $1 \mathrm{keV}$}

In order to reproduce the continuum of all the spectra, we first fitted with the Eastern model of the MCD model (diskbb in XSPEC) and the BB model (bbody in XSPEC), which come from the accretion disk and the NS surface, respectively (Model 1; MCD+BB). We show an example of the result in Figure 3 left, where the NB1 spectrum is displayed with the best fitted model and the residual. The ratios between data and model of all the seven spectra are shown in Figure 3 right. All of the spectra were moderately well described with the MCD+BB model, and the innermost temperature of the disk $\left(T_{\text {in }}\right)$ and the temperature of the NS surface $\left(T_{N S}\right)$ are obtained as $\sim 1.4$ $\mathrm{keV}$ and $\sim 2.2 \mathrm{keV}$, respectively. However, there remained broad excess around $1 \mathrm{keV}$ in all of the spectra. There are also some features around 6-7 keV related to Fe-K lines, and these results are described in other paper (Shirai et al. 2008 in preparation).


Figure 3: Left: A fi tting result of the NB1 spectrum with Model 1 (MCD+ BB). The upper panel shows the data and the model spectra (black: XIS-FI, red: HXD-PIN), and the lower panel is the ratio between them. Right: Ratios between data and model for all the seven spectra fi tted with Model 1. 
One previous XMM-Newton observation of Cyg X-2 estimated the contribution of dust scattering, of which the spectrum has a peak around $1 \mathrm{keV}$ (Constantini et al. 2005). To study the effect, we compare two energy spectra, where one excluded the central 70 pixels around the image core described in section 2 and the other included the central part. Since the photon-pile up does not effect significantly in the soft energy band, we can estimate the contribution of the dust scattering through the comparison. If the $1 \mathrm{keV}$ broad structure is caused by the dust scattering, the spectrum excluding the central 70 pixels should have relatively higher flux, namely the contribution of the dust scattering to the total spectrum is high. Then, We fitted the two spectra simultaneously at the energy range of $0.5-3 \mathrm{keV}$, with the same model of inter-stellar absorption plus MCD, where only the normalization of the MCD component is different. Figure 7 shows the fitting result of the NB1 spectra. It can be seen that both spectra show the same spectra has same amount of the residuals at the low energy band. This result is also the same in the other six spectra. Therefore, the $1 \mathrm{keV}$ broad structure cannot explained only by the effect of the dust scattering.

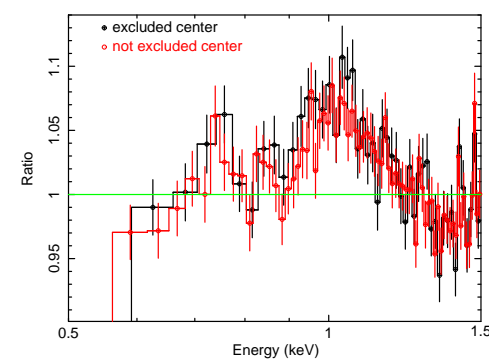

Figure 4: Comparison of spectra which excluded or included the central 70 pixels. Both spectra are fi rtted with Model 1 (MCD+BB).

In the past, this structure was assumed to be a complex of several narrow lines (Di Salvo et al. 2002). We added a broad Gaussian model (Gau) around $1 \mathrm{keV}$ to Model 1 (Model 2; $\mathrm{MCD}+\mathrm{BB}+\mathrm{Gau})$. The results are showed in the left and middle panel of Figure 5. This Model 2 reproduces the broad structure in all the seven spectra. The derived parameters of the Gau model are the center energy of $\sim 0.7 \mathrm{keV}$, width of $\sim 340 \mathrm{eV}$, and equivalent width (EW) of 170-300 eV (Figure 5 right). When this excess is from the complex of several narrow lines, the EW obtained by Suzaku is about one order magnitude larger than the total of narrow lines detected by previous Grating observations (several ten eV; Vrtilek et al. 1988). If this structure comes from a broad line, the estimated line emitting region to make the broad width locates too close to the NS suface ( $35 \mathrm{~km}$ ) to keep such a low ionized state. Therefore, we think neither narrow nor broad lines cannot reproduce the $1 \mathrm{keV}$ structure physically.

We finally added a low-temperature BB model instead of the Gau (Model 3; MCD+BB+BB). The additional BB model can also explain the broad excess, as shown in Figure 6 . The temperature is obtained as constant $\sim 0.2 \mathrm{keV}$ in all of the spectra, and the emitting radius increases with $680-770 \mathrm{~km}$, from NB1 to NB5. The emitting radius, corresponding to $\sim 30$ times larger than the innermost radius of accretion disk. Then, these physical values, the lower temperature and the larger radius compared with those of the disk emission, indicate that the low-temperature BB emission may come from the outer part of the accretion disk.

When the temperature profile of the disk is assumed as $T(r)=T_{i n} \times\left(\frac{r}{r_{i n}}\right)^{-0.75}$, where $T_{\text {in }}$ is tem- 



Figure 5: Left and Middle: Same as Figure 3, but with Model 2 (MCD+BB+Gau). Right: Variabirity of the Gau parameters. From top to bottom, the center energy in $\mathrm{keV}$, the line width in $\mathrm{keV}$ and the line $\mathrm{EW}$ in eV are shown. (the red horizontal line is average value of each parameters)

perature of inner radius, $r_{i n}$ is the innermost radius of the disk and $r$ is distance from the innermost radius, the temperature at $r \sim 680 \mathrm{~km}\left(T_{\text {in }}=1.44 \mathrm{keV}\right.$ and $r_{i n}=24 \mathrm{~km}$ in the NB5) is estimated as $0.12 \mathrm{keV}$, which is close to that of the low-temperature BB. When the accretion disk become to be the slim disk, the distribution of temperature profile is predicted to become $p<0.75$, making the flux in the soft energy band relatively higher than that in the hard band. Therefore, the current result of the additional BB component in the lower energy band, may be consistent with the picture of the slim disk.
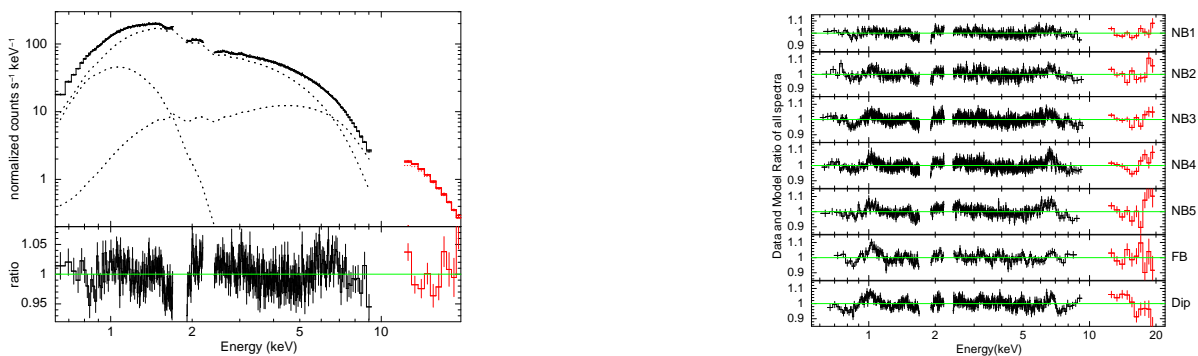

Figure 6: Same as Figure 3, but with Model $3(\mathrm{MCD}+\mathrm{BB}+\mathrm{BB})$.

From the above results, the $1 \mathrm{keV}$ excess can be explained by neither the dust scattering nor the broad/narrow Gaussian emission but the lower temperature BB component, which may be caused by the change of the emissivity according to the disk state from standard to slim.

\section{References}

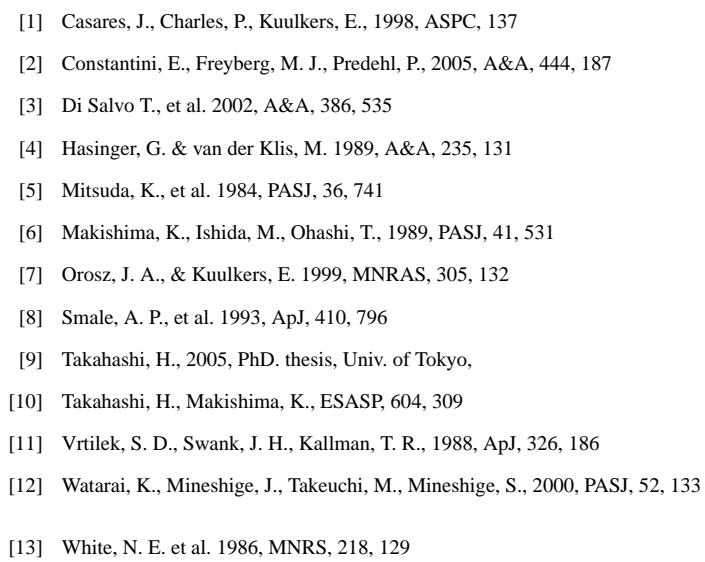

